Diabetologe 2015 • 11:112-113

DOI 10.1007/s11428-014-1284-7

Online publiziert: 21. März 2015

(c) Springer-Verlag Berlin Heidelberg 2015

\title{
R. Landgraf
}

Deutsche Diabetes-Stiftung, München

\section{Diabetisches Fußsyndrom}

Beteiligung so vieler verschiedener Fachdisziplinen über alle Sektoren der Versorgung hinweg erforderlich macht wie das DFS. Die Betreuung von DFS-Patienten ist sehr zeitaufwendig, inakzeptabel viel mit Bürokratie verbunden, kaum leistungs- und ergebnisorientiert vergütet, wird nicht selten von unerfahrenen Fachkollegen bewerkstelligt und mündet bisweilen sogar in Kompetenzgerangel.

\section{I) Netzwerke sind der Schlüssel für den Erfolg}

Amputationsraten durch adäquate Therapie und Kooperationen bereits signifikant vermindert werden. Wird jedoch die Zahl der Amputationen in Deutschland der letzten 25 Jahre betrachtet, muss festgestellt werden, dass flächendeckend die Gesamtzahl an Amputationen eher zugenommen hat. Majoramputationen haben jedoch im Zeitraum zwischen 2005 und 2010 um 15\% bei Männern und um 27\% bei Frauen erfreulicherweise abgenommen. Dies betrifft v. a. die über 80-Jährigen, bei denen die Häufigkeit von Majoramputationen sogar um 30\% reduziert werden konnte. Dagegen haben die Minoramputationen bei Männern um 47\% zugenommen. Dies ist nicht so enttäuschend wie es zunächst scheint, da diese Maßnahmen häufiger vorgenommen werden, um den Heilungsverlauf und die Phase der Immobilisierung bei solchen Patienten abzukürzen.

Warum gelingt es nur in wenigen Zentren und Regionen in Deutschland eine bessere Betreuung von Menschen mit DFS umzusetzen und damit die Amputationsraten zu senken? Prävention und Versorgung des DFS sind nur möglich durch vertrauensvolle, kompetente und interdisziplinäre Kooperation. Es gibt keine Komplikation des Diabetes, die eine
Die Instrumente für eine optimale Behandlung sind vorhanden: Dazu gehören evidenzbasierte Nationale Versorgungsleitlinien, Disease-Management-Programme (DMP) mit klarer Schnittstellenregelung sowie eine aktive Arbeitsgemeinschaft (AG Fuß der Deutschen Diabetes Gesellschaft). Diese beschäftigt sich u. a. mit der Entwicklung eines Qualitätsmanagementsystems, der Zertifizierung von Arztpraxen und Kliniken als spezialisierte Fußeinrichtungen sowie mit der speziellen Ausbildung von Podologen, Fußschwestern und Wundmanagern. Außerdem ist die AG Fuß mit der Bildung von Netzwerken wie das Fußnetz Köln, als Vorreiter und Modell, und viele anderen Aktivitäten betraut.

Netzwerke sind grundlegend wichtig, sie führen die relevanten Leistungserbringer einer Region zusammen. Mit ihnen können abgestimmte Behandlungsund Präventionskonzepte für Patienten mit DFS erarbeitet werden. Darüber hinaus steigern sie die Qualität durch Herstellung von Transparenz mittels offenem Benchmarking (Schwere der Erkrankung und Ergebnisse der Behandlung) und Hospitation sowie kollegialem Austausch in Qualitätszirkeln. Die Beteiligung der Kostenträger ist bei der Entwicklung solcher Netzwerke integraler Bestandteil im Sinne einer Qualitätspartnerschaft.

In dem vorliegenden Heft wird der aktuelle Stand in der Betreuung und Rezidivprophylaxe des DFS diskutiert. Eine Versorgungsforschung ist v. a. dann möglich, wenn eine große Anzahl unterschiedlich ausgeprägter DFS-Fälle strukturiert und validiert dokumentiert werden, und zwar vor, während und langfristig nach der Behandlung eines DFS. Im Beitrag von Risse et al. wird ein national ausgelegtes, über viele Fachbereiche hinweg konsentiertes DFS-Register vorgestellt, das mit einem umfangreichen Datensatz helfen kann, die wichtigsten Probleme zur Prophylaxe und Behandlung des meist multimorbiden Menschen mit DFS zu lösen. Die Ziele des DFS-Registers sind:

- epidemiologische Datenerhebung,

- strukturierte Datenerfassung und externe Qualitätskontrolle,

- Etablierung eines bundesweiten Registers, das hilft, die Versorgungskette(n) abzubilden, die Kosten zu erfassen und gesundheitsökonomische Auswirkungen zu analysieren.

Dabei werden die Daten im Rahmen der Dokumentation von Selektivverträgen mit Kostenträgern in verschiedenen Endbenutzersoftwareprogrammen erhoben, geprüft und quartalsweise exportiert. Die Datensammelstelle leitet sie an berechtigte Institutionen weiter. Sie pflegt das Prüfmodul für die Endbenutzersoftwareprogramme und betreut die Dokumentierenden. Vertreter von Krankenkassen, verschiedenen Fachgesellschaften und bisher in Selektivverträgen dokumentierenden Einrichtungen haben damit ein weltweit einzigartiges Instrument zur Doku- 
mentation und Qualitätssicherung des DFS etabliert.

Raabe beschreibt in seinem Beitrag die wichtigsten medizinischen und organisatorischen Aufgaben bei der Erstversorgung von Menschen mit DFS. Dabei stellt die strukturierte multidisziplinäre Erstversorgung die wesentliche Basis für Langzeitergebnisse beim DFS dar.

Hochlenert et al. analysieren mithilfe eines Entitätenkonzepts die Lokalisationen des DFS hinsichtlich typischer Ursachen. Die Entität beschreibt ein relativ homogenes Krankheitsbild mit definierter Lokalisation und Pathobiomechanik, der Wahrscheinlichkeit einer peripheren arteriellen Verschlusskrankheit (PAVK) und anderen Ursachen sowie geeigneten Entlastungsmöglichkeiten und prognostischen Informationen.

Außerdem zeigen Jecht et al. in ersten Analysen Ergebnisse des DFS-Registers mit wesentlichen Charakteristika von Menschen mit dieser Krankheit. Es ist wohl die weltweit größte Sammlung strukturiert und gerichtet erhobener Daten zu DFS: Insgesamt 102 Einrichtungen haben bei 25.038 Diabetespatienten 57.847 DFS-Fälle dokumentiert. Dies ermöglicht, Longitudinalverläufe einzelner Phasen der Erkrankung auch phasenübergreifend zu analysieren. Dabei finden sich geschlechts- und altersgruppenabhängige Unterschiede bezüglich

- des Behandlungsbeginns,

- des Vorliegens einer PAVK,

- der Pflegebedürftigkeit,

- der Nierenersatztherapie und

- des Charcot-Fußes.

Alle Autoren wünschen sich viele Leser und großes Interesse beim Studieren der praxisrelevanten Beiträge zum DFS. Die Publikationen wurden von Wissenschaftlern und Teams verfasst, die über umfangreiche praktische Erfahrungen mit dem DFS verfügen und nicht nur nationales, sondern auch internationales Renommee genießen. Die Beteiligung von immer mehr Fachkollegen am DFS-Register und die Umsetzung des Entitätenkonzeptes würde die Versorgung von Menschen mit DFS wesentlich stärken und verbessern sowie dazu beitragen, dass sich in unserem Lande endlich flächendeckend die schwerwiegenden Folgen eines DFS reduzieren lassen und deutlich mehr Rezidive vermieden werden.

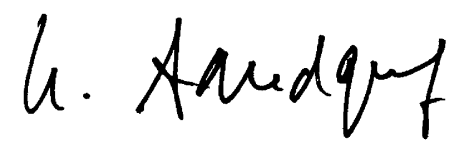

R. Landgraf

\section{Korrespondenzadresse}

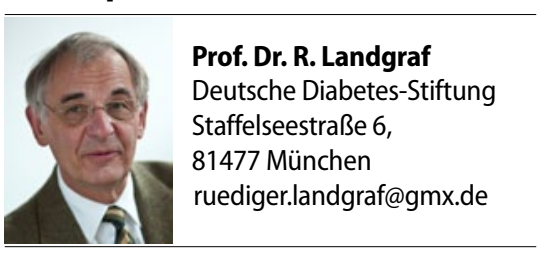

Interessenkonflikt. R. Landgraf gibt an, dass kein Interessenkonflikt besteht.

\section{Entwicklung von Kindern und Jugendlichen}

KiGGS: Welle 1

Die Entwicklung von Kindern und Jugendlichen sowie ihre Chancen auf ein Leben

in guter Gesundheit hängen nicht nur von körperlichen und psychischen Faktoren ab, sondern werden auch durch das familiäre und soziale Umfeld geprägt. Diese Zusammenhänge untersucht die erste Folgeerhebung (KiGGS Welle 1) der "Studie zur Gesundheit von Kindern und Jugendlichen in Deutschland" (KiGGS).

Im Schwerpunktheft „Studie zur Gesund-

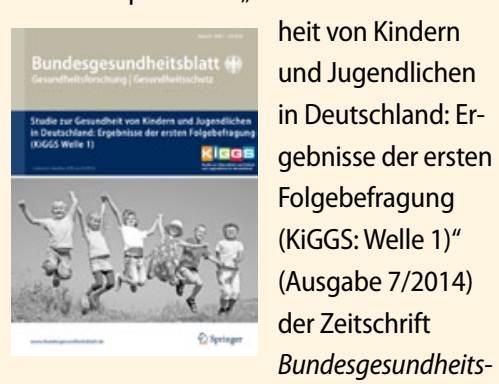

blatt werden u.a. folgende wichtige Daten, Analysen und Trendentwicklungen aus der neuen KiGGS-Befragungswelle vorgestellt:

— Chronische Erkrankungen und impfpräventable Infektionserkrankungen

- Tabak- und Alkoholkonsum bei 11- bis 17-jährigen Jugendlichen

- Einflussfaktoren auf Verbreitung und Dauer des Stillens in Deutschland

- Körperlich-sportliche Aktivität und Nutzung elektronischer Medien im Kindesund Jugendalter

Bestellen Sie diese Ausgabe zum Preis von 16,- EUR zzgl. Versandkosten bei Springer Customer Service Center Kundenservice Zeitschriften

Haberstr. 7

69126 Heidelberg

Tel.: +49 6221-345-4303

Fax: +49 6221-345-4229

E-Mail: leserservice@springer.com

Suchen Sie noch mehr zum Thema? Mit e.Med, dem Online-Paket von Springer Medizin, können Sie schnell und komfortabel in über 600 medizinischen Fachzeitschriften recherchieren. Weitere Infos unter springermedizin.de/eMed. 